Chapman University

Chapman University Digital Commons

Pharmacy Faculty Articles and Research

School of Pharmacy

$1-9-2012$

\title{
Synthetic Peptides Derived from the Sequence of a Lasso Peptide Microcin J25 Show Antibacterial Activity
}

Rania Soudy

University of Alberta

Liru Wang

CanBiocin Inc.

Kamaljit Kaur

ChapmanUniversity, kkaur@chapman.edu

Follow this and additional works at: https://digitalcommons.chapman.edu/pharmacy_articles

Part of the Amino Acids, Peptides, and Proteins Commons, Chemical and Pharmacologic

Phenomena Commons, Medical Biochemistry Commons, Medicinal and Pharmaceutical Chemistry

Commons, and the Other Pharmacy and Pharmaceutical Sciences Commons

\section{Recommended Citation}

Soudy R, Wang L, Kaur K. Synthetic peptides derived from the sequence of a lasso peptide microcin J25 show antibacterial activity. Bioorg. Med. Chem. 2012;20(5):1794-1800. doi: 10.1016/j.bmc.2011.12.061

This Article is brought to you for free and open access by the School of Pharmacy at Chapman University Digital Commons. It has been accepted for inclusion in Pharmacy Faculty Articles and Research by an authorized administrator of Chapman University Digital Commons. For more information,

please contact laughtin@chapman.edu. 


\section{Synthetic Peptides Derived from the Sequence of a Lasso Peptide Microcin J25 Show Antibacterial Activity}

\section{Comments}

NOTICE: this is the author's version of a work that was accepted for publication in Bioorganic \& Medicinal Chemistry. Changes resulting from the publishing process, such as peer review, editing, corrections, structural formatting, and other quality control mechanisms may not be reflected in this document. Changes may have been made to this work since it was submitted for publication. A definitive version was subsequently published in Bioorganic \& Medicinal Chemistry, volume 20, issue 5, in 2012. DOI: 10.1016/j.bmc.2011.12.061

The Creative Commons license below applies only to this version of the article.

\section{Creative Commons License}

\section{(c) 1 (1) 90}

This work is licensed under a Creative Commons Attribution-Noncommercial-No Derivative Works 4.0 License.

\section{Copyright}

Elsevier 


\title{
Synthetic peptides derived from the sequence of a
}

\section{lasso peptide microcin $\mathrm{J} 25$ show antibacterial}

\author{
activity
}

Rania Soudy, ${ }^{+}$Liru Wang, ${ }^{\dagger}$ and Kamaljit Kaur ${ }^{t, *}$

Faculty of Pharmacy and Pharmaceutical Sciences, University of Alberta, Edmonton, Alberta, Canada, T6G 2N8, and ${ }^{\dagger}$ CanBiocin Inc., Edmonton, Alberta T5J 4P6

kkaur@ualberta.ca

RECEIVED DATE (to be automatically inserted after your manuscript is accepted if required according to the journal that you are submitting your paper to)

Tel. 780-492-8917; Fax. 780-492-1217; email. kkaur@ualberta.ca 


\begin{abstract}
Microcin J25 (MccJ25) is a plasmid-encoded, ribosomally synthesized antibacterial peptide with a unique lasso structure. The lasso structure, produced with the aid of two processing enzymes, provides exceptional stability to MccJ25. We report the synthesis of six peptides (1-6), derived from the MccJ25 sequence, that are designed to form folded conformation by disulfide bond formation and electrostatic or hydrophobic interactions. Two peptides (1 and 6) display good activity against Salmonella newport, and are the first synthetic derivatives of MccJ25 that are bactericidal. Peptide 1 displays potent activity against several Salmonella strains including two MccJ25 resistant strains. The solution conformation and the stability studies of the active peptides suggest that they do not fold into a lasso conformation and peptide $\mathbf{1}$ displays antimicrobial activity by inhibition of target cell respiration. Like MccJ25, the synthetic MccJ25 derivatives display minimal toxicity to mammalian cells suggesting that these peptides act specifically on bacterial cells.
\end{abstract}

Keywords

lasso peptide; microcin J25 (MccJ25); synthetic MccJ25 derivatives; antimicrobial activity 


\section{Introduction}

Microcin J25 (MccJ25) is a 21-residue ribosomally synthesized antimicrobial peptide that adopts a remarkable threaded-lasso structure. ${ }^{1-3}$ MccJ25 displays bactericidal activity against a range of food-borne disease-causing Gram-negative pathogens including diarrheagenic E. coli strains. ${ }^{4}$ The lasso-structure of the peptide consists of a lactam ring (known as lariat ring) formed between the N-terminus Gly1 and the side chain of Glu8 (Figure 1) ${ }^{5-7}$ The C-terminal tail (Tyr9-Gly21) passes through the ring forming a $\beta$-hairpin structure made of a type I $\beta$-turn (Val11-Gly14) and two short double stranded antiparallel $\beta$-sheets, residues 6-7/19-20 and 1011/15-16.

The active mature MccJ25 is produced and exported by four plasmid-encoded genes $m c j A B C D{ }^{8}$ McjA encodes a linear 58-residue precursor peptide which is converted into mature peptide by two enzymes, $\mathrm{McjB}$ and $\mathrm{McjC} .{ }^{9}{ }^{10} \mathrm{McjB}$, a putative peptidase, cleaves the 37-residue leader peptide from the precursor peptide, whereas, $\mathrm{McjC}$ is believed to catalyze the lactam bond formation. McjD helps in the transport of the active antibiotic peptide out of the producing cell. ${ }^{11}$ The lasso-structure confers several properties to $\mathrm{MccJ} 25$, such as, resistance to proteolytic degradation, ${ }^{12,13}$ stability towards chaotropes, organic solvents, and extreme temperatures. ${ }^{14}$ Such properties make MccJ25 a potential candidate for a number of applications including food preservation and treatment of food-borne diseases.

Different variants of MccJ25, prepared by site directed mutagenesis, have been reported for studying structure activity relationship (SAR). ${ }^{15}, 16$ Pavlova et al. performed a complete mutational scanning analysis of MccJ25 by making more than $380 \mathrm{MccJ} 25$ variants with a single amino acid substitution. ${ }^{16}$ Each single amino acid variant was tested for production and export from E.coli. In addition, the variants that were produced were analyzed for the ability to inhibit 
RNA polymerase (RNAP) and bacterial growth. Several residues in the lactam ring and the tail region were found to be important for the production of MccJ25 and inhibition of bacterial growth. Another study reported MccJ25 variants containing two or three amino acid substitutions where the peptides retain the lasso structure and the antimicrobial function. ${ }^{15}$ These studies demonstrate that peptide lasso scaffold of $\mathrm{MccJ} 25$ is quite tolerant to amino acid substitutions. Additional SAR studies of MccJ25 have suggested that the lactam ring region and the tail $\beta$-hairpin loop play distinct roles in the peptide's antimicrobial activity. ${ }^{17,18}$ In particular, using a thermolysin-digested MccJ25 cleaved between residues 10 and 11 it was demonstrated that the $\beta$-hairpin (residues 10-16) is crucial in the import of MccJ25 through FhuA, but not for RNAP and respiration inhibition. ${ }^{18}$ The roles played by specific residues in the peptide have also been reported. ${ }^{19,} 20$ The C-terminal glycine was found to be important for RNAP inhibition, ${ }^{19}$ while site specific mutation of His-5 demonstrated that this residue was important for recognition by the inner membrane receptor SbmA..$^{20}$

Attempts have also been made to chemically synthesize MccJ25 variants, such as, macrocyclic head-to-tail 21-residue MccJ25, a 21-residue variant with side chain-to-backbone lactam ring between $\alpha$-amino of $\mathrm{Gly}^{1}$ and $\gamma$-carboxyl of $\mathrm{Glu}^{8}$, and a recently reported MccJ25 model peptide (cMccJ25) where $\mathrm{Gly}^{1}$ and $\mathrm{Glu}^{8}$ are replaced with nearly isosteric azidoacetic acid and propargyl glycine, respectively. ${ }^{5,6,21}$ In the later variant (cMccJ25), the copper-catalyzed click reaction between the azide and the terminal alkyne permits cyclization of $\mathrm{cMccJ} 25$ without the influence of the maturation enzymes. ${ }^{21}$ None of these synthetic peptides showed any antibacterial activity.

We hypothesized that peptides with antibacterial activity can be designed based on the MccJ25 sequence where a folded conformation is acquired by a combination of intra-peptide 
disulfide bond formation and electrostatic or hydrophobic interactions, instead of posttranslational modification by McjB and McjC proteins. To test our hypothesis, six peptides (1-6) derived from the sequence of MccJ25 ranging in length 18-22 residues were designed and synthesized (Figure 1). We report herein the results of antimicrobial activity, solution conformation, stability to proteases and cytotoxicity of this series of synthetic MccJ25 derivatives.

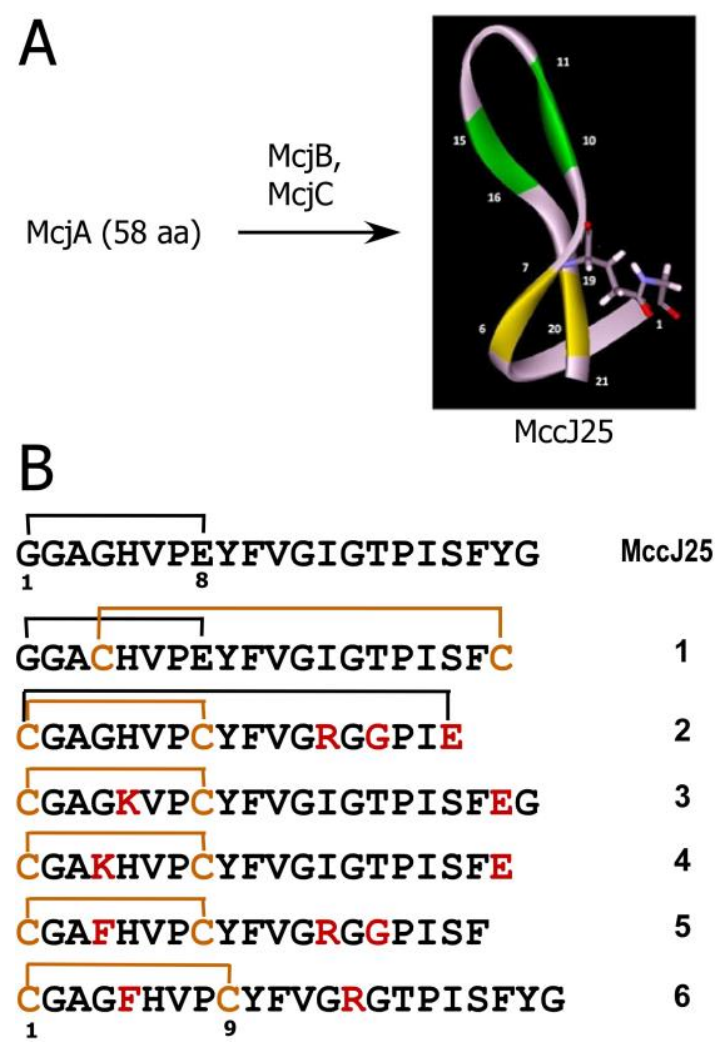

Figure 1. (A) A 58 amino acid McjA precursor peptide is converted into mature antimicrobial form, MccJ 25 by McjB and McjC enzymes. The ribbon representation of the lasso structure of MccJ25 (PDB ID code 1Q71) ${ }^{6}$ is shown. Antiparallel $\beta$-sheets are shown in green and yellow, and the amide bond between residues G1-E8 is shown as stick model. (B) Amino acid sequences of MccJ25 and derived peptides 1-6. Substitutions of amino acids in the peptide derivatives are shown in yellow for cysteine and red for others. 


\section{Results and Discussion}

Six peptides were designed on the basis of the MccJ25 sequence following the strategy that intra-peptide interactions will lead to active peptide derivatives. Peptides were designed to form folded conformation by intra-peptide (i) disulfide and amide bond formation (analogues $\mathbf{1}$ and 2), (ii) disulfide and electrostatic interactions (3 and 4), or (iii) disulfide and hydrophobic interactions (5 and 6). In peptide 1, the lactam ring of MccJ25 between G1 and E8 was maintained, and a disulfide bond between C4 and C20 (C-terminal) was introduced to hold the C-terminal of the peptide in place. In all other peptides (2-6), the isopeptide bond between G1 and E8 was replaced with a disulfide bond. Peptide 2, an 18-residue peptide, utilized an amide bond to tie the $\mathrm{N}$-terminal $\mathrm{C} 1$ with the $\mathrm{C}$-terminal E18. Peptides $\mathbf{3}$ and $\mathbf{4}$ contain substitution of a ring residue and one residue from the tail region with positively and negatively charged amino acids, respectively, for electrostatic interactions (H5K, Y20E in $\mathbf{3}$ and G4K, Y20E in 4). Similarly, peptides 5 and $\mathbf{6}$ contain substitution or insertion with hydrophobic amino acids, such as G4F in 5. Peptide 6 consists of a larger 9-residue N-terminal ring, as expansion of the ring was thought to facilitate the insertion of the $\mathrm{C}$-terminal into the $\mathrm{N}$-terminal lariat ring by interaction between F5 and F19 and/or Y20. An aromatic hydrophobic Phe (F) residue was inserted between G4 and H5 for enhanced van der Waals and stacking interactions with the Cterminal F19 and Y20. In addition, I13R and/or T15G substitutions (replacement of hydrophobic Ile with basic guanidine containing Arg and Thr with a methyl side chain with Gly with no side chain) were introduced in peptides $\mathbf{2 , 5}$, and $\mathbf{6}$ to increase solubility. These mutations have been reported to yield higher inhibitory activity compared to the wild-type MccJ25. ${ }^{16}$ 
Peptides 1-6 were synthesized as linear peptides using Fmoc solid-phase peptide synthesis. For peptides $\mathbf{1}$ and $\mathbf{2}$, the lactam bond formation was achieved on the solid-phase, followed by cleavage from the resin. The second ring (disulfide bond) was then formed in solution. Peptides 3-6 were cleaved from the resin as linear peptides followed by oxidation to form disulfide bond. The electrostatic or hydrophobic interactions in the peptides were initiated when the peptides were cleaved from the solid phase and the side chain protections were removed. Native MccJ25 was expressed using pTUC202 plasmid in E. coli MC4100 in M9 minimal media. ${ }^{12}$ Crude peptides were purified using semi-preparative reversed-phase HPLC prior to characterization by electrospray and/or MALDI-TOF mass spectrometry (Table S1, supplementary data). All peptides were purified up to $>95 \%$ purity as shown by analytical RPHPLC and mass spectrometry (Figures S1 and S2), and were obtained with an overall yield of 7$32 \%$.

The antibacterial activity of MccJ25 and peptides 1-6 was evaluated against a number of Gram-negative pathogens. Twelve Salmonella strains and two E. coli strains were used to obtain the activity profile of the peptides using liquid growth inhibition assay (Table 1). ${ }^{22}$ Peptides $\mathbf{1}$ and 6 displayed good activity (low micromolar range) against S. newport with MIC values of 25 and $30 \mu \mathrm{M}$, respectively (Figure 2). Compared to the native MccJ25 (MIC $0.5 \mu \mathrm{M}$ ), these peptides were 50-60 fold less potent. Interestingly, 1 also displayed activity against several (five) other Salmonella strains including two MccJ25 resistant strains. For all of these strains, MIC of 1 was in the range of 75-90 $\mu \mathrm{M}$. The MIC of MccJ25 was also higher (compared to $S$. newport) for the three MccJ25 sensitive strains. Peptides 1-6 were also tested for inhibition of Gram-positive bacteria, such as S. aureus ATCC 6538 and E. faecalis ATCC 19433. These peptides showed no activity against Gram-positive strains. 
A number of different MIC values of MccJ25, ranging from $5 \mathrm{nM}$ to $0.22 \mu \mathrm{M}$, against $S$. newport have been reported previously. ${ }^{3,12,23,24}$ In this study, we found the MIC value of MccJ25 to be $0.5 \mu \mathrm{M}$ against a clinical isolate of $S$. newport. This variation in MIC values could be due to the difference in the experimental conditions used, such as spot-on-lawn assay, liquid growth assay, media with or without supplements, or the difference in the strains used.

Table 1. Antibacterial activity of MccJ25 and synthetic peptide derivatives (1 and $\mathbf{6}$ ) against Gram-negative bacterial strains

\begin{tabular}{|c|c|c|c|}
\hline \multirow{2}{*}{ Strains $^{\mathbf{a}}$} & \multicolumn{3}{|c|}{ MIC $^{b}(\mu M)$ of Peptides } \\
\hline & MccJ25 & 1 & 6 \\
\hline S. newport & $0.5 \pm 0.05$ & $25 \pm 3.0$ & $30 \pm 4.0$ \\
\hline S. enteritidis PT8 & $1.5 \pm 0.8$ & $80 \pm 3.0$ & - \\
\hline S. enteritidis ATCC13076 & $1.8 \pm 0.7$ & - & - \\
\hline S. enteritidis AFLB 11 & $2.0 \pm 0.6$ & - & - \\
\hline S. enteritidis AFLB 41 & $1.6 \pm 1.0$ & - & - \\
\hline S. enteritidis AFLB 81 & $1.7 \pm 1.2$ & - & - \\
\hline S. typhimurium AFLB 25 & $2.8 \pm 0.8$ & $85 \pm 5.2$ & - \\
\hline S. typhimurium ATCC 13311 & - & $75 \pm 6.5$ & - \\
\hline S. heidelberg ATCC 8326 & - & $83 \pm 6.1$ & - \\
\hline S. montevideo 20 & - & - & - \\
\hline S. paratyphi & $1.5 \pm 1.0$ & - & - \\
\hline S. choleresius ATCC 10708 & $1.5 \pm 0.6$ & $90 \pm 5.7$ & - \\
\hline E. coli DH5 $\alpha$ & $5.0 \pm 1.0$ & - & - \\
\hline E. coli MC4100 & $5.0 \pm 2.0$ & - & - \\
\hline \multicolumn{4}{|c|}{$\begin{array}{l}{ }^{a} \text { Details for the bacterial strains are provided in Table } \mathrm{S} 2 \text { (supplementary data). } \\
{ }^{\mathrm{b}} \mathrm{MIC} \text { value is the last concentration of the peptide }(\mu \mathrm{M}) \text { giving } 100 \% \text { inhibition } \\
\text { of the strain. -, no activity detected up to } 250 \mu \mathrm{M} \text {. Data are represented as } \\
\text { means } \pm \text { standard errors of the means. The values are results of at least three } \\
\text { independent measurements. }\end{array}$} \\
\hline
\end{tabular}




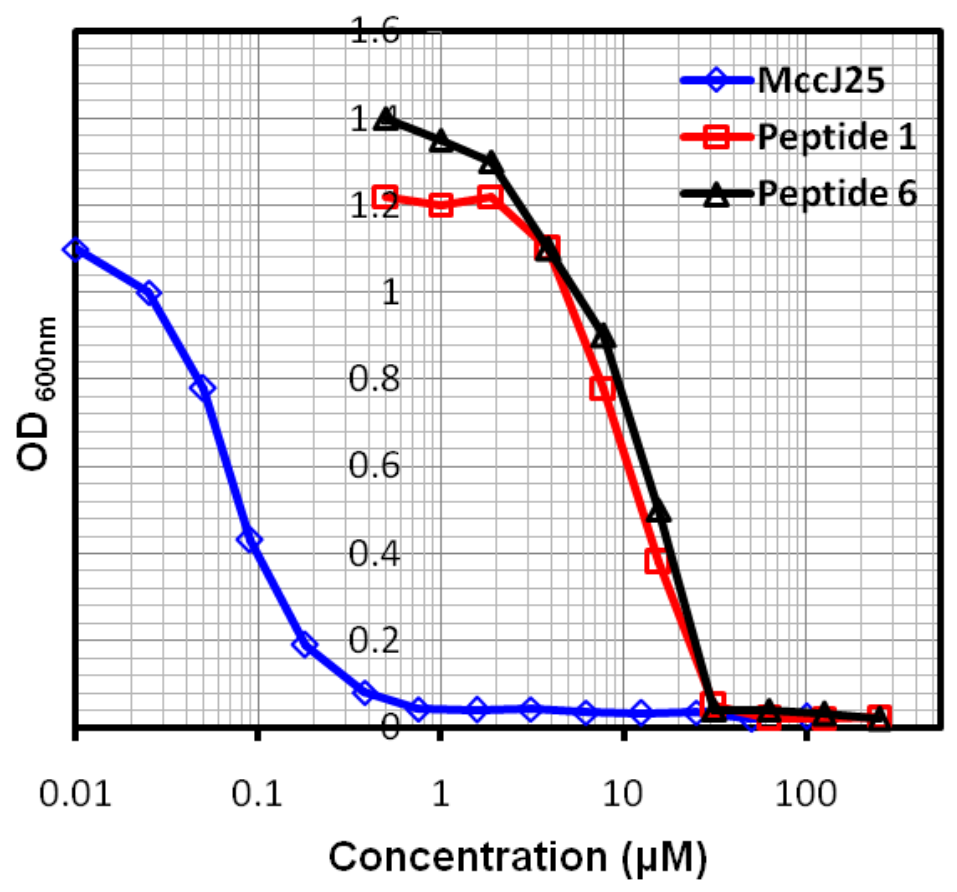

Figure 2. Growth curves of microcin J25, peptide 1, and peptide $\mathbf{6}$ using broth assay against Salmonella newport. MICs were defined as the lowest peptide concentration that caused $100 \%$ growth inhibition (0.05 AU).

Furthermore, peptides 1 and $\mathbf{6}$ were found to be bactericidal similar to MccJ25. ${ }^{4}$ The minimum bactericidal concentrations (MBCs) of peptides $\mathbf{1}$ and $\mathbf{6}$ against $S$. newport were 31.25 and $62.5 \mu \mathrm{M}$, respectively. The MBCs of peptide 1 against $S$. enteritidis ATCC P76, $S$. typhimurium ATCC 13311, and S. choleresius ATCC 10708 were $125 \mu \mathrm{M}$ each. A number of synthetic analogues of MccJ25 have been reported, however, none of them display activity against Gram-negative pathogens. ${ }^{6,7,13,21}$ To our knowledge, peptides $\mathbf{1}$ and $\mathbf{6}$ are the first synthetic derivatives of MccJ25 that display antibacterial activity. Peptides 2-5 lacked antimicrobial activity against all of the tested strains up to $250 \mu \mathrm{M}$ concentration. One striking 
feature of peptides 2-5 was the replacement of lactam bond with a disulfide linkage. Although the same is true for peptide $\mathbf{6}$, but the replacement was better tolerated due to the expanded 9residue lariat ring. A variant of peptide $\mathbf{6}$ with 8-residue lactam ring (CGAFHVPCYFVGRGTPISFYG) was synthesized (not shown here) and was found to be inactive suggesting larger lactam ring facilitates interaction between the C-terminal tail and the $\mathrm{N}$-terminal ring. The reduced activity of $\mathbf{1}$ and $\mathbf{6}$ compared to the native MccJ25 could be due to the loss of characteristic lasso conformation.

Circular dichroism (CD) spectroscopy was used to study the folding behavior of peptides 1 and $\mathbf{6}$ in solution. Methanol was used as a solvent for CD studies due to the limited solubility of the peptides in water. Moreover, methanol has been used previously to elucidate the solution structure (NMR structure) of MccJ25. ${ }^{6}$ The CD spectra of peptides $\mathbf{1}$ and $\mathbf{6}$ in methanol were very different from MccJ25 suggesting different folded structures in solution (Figure 3). The CD spectrum of MccJ25 in methanol displayed a minimum at $200 \mathrm{~nm}\left(\Theta=-3.2 \times 10^{3}\right)$ and a maximum at $225 \mathrm{~nm}\left(\Theta=1.1 \times 10^{3}\right)$ with a positive shoulder at $215 \mathrm{~nm}$ (Figure 3) consistent with the previously reported spectrum. ${ }^{25}$ A negative peak at $200 \mathrm{~nm}$ is characteristic of unstructured peptides; however, this peak has also been attributed to small $\beta$-sheets or $\beta$-turn secondary structures such as in $\beta$-defensins. ${ }^{26}$ The positive band at ca. 225 and $210 \mathrm{~nm}$ have been attributed to the Phe $\mathrm{L}_{\mathrm{a}}$ and Tyr $\mathrm{L}_{\mathrm{a}}$ transitions. ${ }^{27} \mathrm{CD}$ of peptide $\mathbf{1}$ showed a broad minimum at $208 \mathrm{~nm}$ with low intensity $\left(\Theta=-1.5 \times 10^{3}\right)$, whereas, peptide 6 displayed a minimum at 210 $\mathrm{nm}\left(\Theta=-5.0 \times 10^{3}\right)$ with a shoulder at $226 \mathrm{~nm}\left(\Theta=-1.8 \times 10^{3}\right)$. Quantitative analysis of the secondary structure of peptide $\mathbf{6}$ with $\mathrm{CDPro}^{28}$ suggested that there is little $\beta$-sheet conformation ( $\sim 15 \%$ residues form $\beta$-sheet) compared to MccJ25 (39\%). Peptide 6 showed the presence of 
increased helical folding. These results suggest that both the active peptides ( $\mathbf{1}$ and $\mathbf{6})$ may adopt a constrained and compact folded structure, however, do not adopt the true lasso structure as observed in MccJ25.

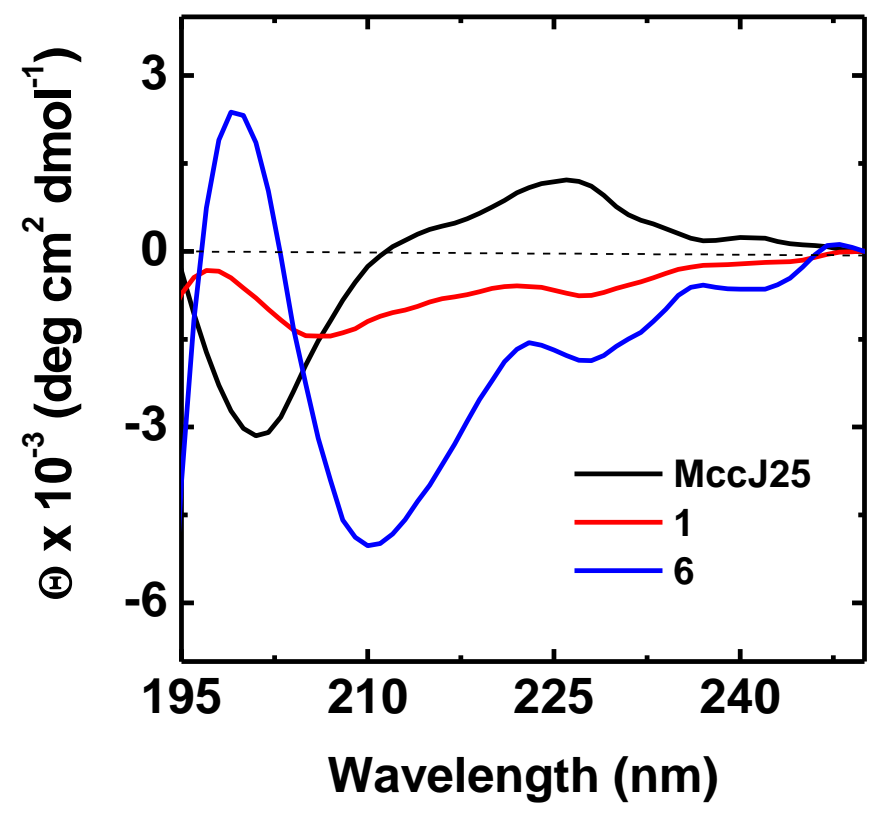

Figure 3. Circular dichroism spectra for $\mathrm{MccJ} 25, \mathbf{1}$ and $\mathbf{6}$ in methanol at $25^{\circ} \mathrm{C}$.

Furthermore, the absence of lasso conformation in peptides $\mathbf{1}$ and $\mathbf{6}$ was confirmed by enzyme stability studies. MccJ25 is known to be highly stable to degradation in the presence of proteolytic enzymes, such as chymotrypsin, trypsin, carboxypeptidase and pepsin. ${ }^{12}$ In contrast, both 1 and 6 were not stable in the presence of chymotrypsin and pepsin. During the antimicrobial assay, the circular growth inhibition zones of $\mathbf{1}$ and $\mathbf{6}$ were distorted to a crescentshape in the presence of a drop of chymotrypsin. Likewise, reversed-phase HPLC analysis of 
peptides 1 and $\mathbf{6}$ incubated with pepsin for 30 minutes showed complete degradation of the peptides (Figure 4). For peptide 1, the presence of fragments FVGIGTPIS and FVGIGTPISF showed that the cleavage occurred before and after the C-terminal Phe residue (Phe19). This suggests that the $\mathrm{C}$-terminal cysteine is present outside the ring ( $\mathrm{N}$-terminal ring between Gly1 and Glu8) allowing access to the C-terminal residues for cleavage by enzymes such as pepsin. Peptide 6 also gave similar degradation products after incubation with pepsin. However, fragments from cleavage near Phe5 residue, which is present inside the N-terminal ring (between Cys 1 and Cys9) of $\mathbf{6}$, were not detected. Interestingly, the evaluation of thermostability of $\mathbf{1}$ and $\mathbf{6}$ showed that only peptide $\mathbf{1}$ was active after incubation in boiling water for 3 hours. This may be due to its rigid bicyclic ring structure allowing high heat resistance similar to wild-type MccJ25. The disulfide bond was found to be intact for peptide $\mathbf{1}$ after boiling treatment. Peptides 1 and $\mathbf{6}$ with reduced cysteine residues (absence of disulfide bond) were found to display no antimicrobial activity. 

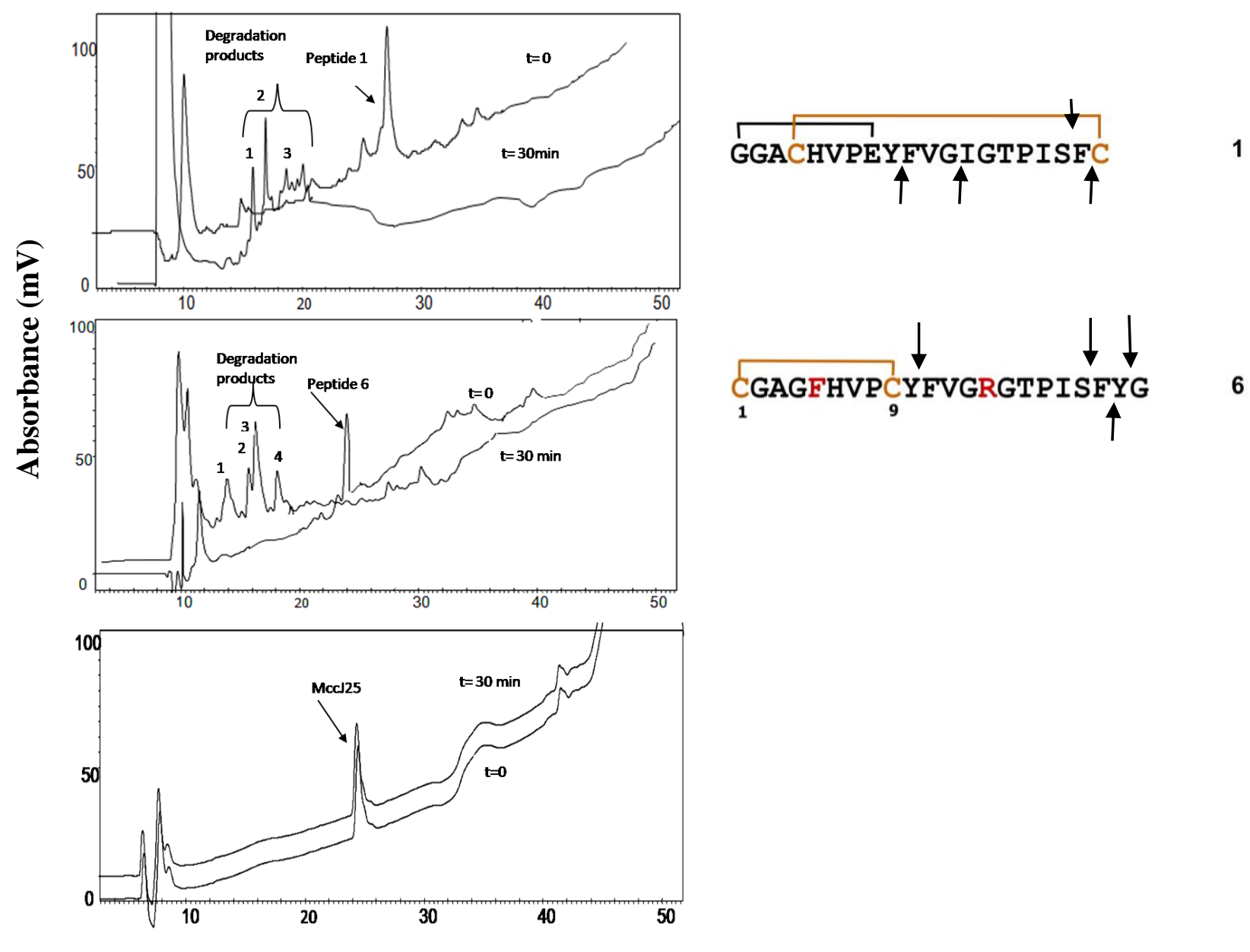

Time (min)

\begin{tabular}{cll}
\hline Fragment \# & \multicolumn{1}{c}{ Sequence } & \multicolumn{1}{c}{ Mass Found } \\
\hline Peptide $\mathbf{1}$ & & \multicolumn{1}{c}{889.1 and 1036.5} \\
1 & FVGIGTPIS and FVGIGTPISF & 1034.6 and 1181.2 \\
2 & GGACHVPEY-C and GGACHVPEY-FC & 1748.8 \\
3 & GGACHVPEY-IGTPISFC & \multicolumn{2}{c}{932.2} \\
\hline Peptide $\mathbf{6}$ & & 1052.1 \\
1 & FVGRGTPIS & 1079.2 and 1242.6 \\
2 & CGAGFHVPCY & $1966.9,2112.9$ and 2276.0 \\
3 & FVGRGTPISF and FVGRGTPISFY & \\
4 & CGAGFHVPCYFVGRGTPIS, \\
& CGAGFHVPCYFVGRGTPISF, and & \\
\hline
\end{tabular}

Figure 4. RP-HPLC chromatograms of peptides 1 (top), 6 (middle) and MccJ25 (bottom) after incubation with pepsin at time 0 and $30 \mathrm{~min}$. On the right side are shown the cleavage sites for the peptides. A linear gradient was run on a C18 Vydac column $(4.6 \mathrm{~mm} \times 250 \mathrm{~mm})$ over 35 min from 15 to $50 \%$ isopropyl alcohol/water $0.05 \%$ (v/v) TFA with a flow rate of $2 \mathrm{~mL} / \mathrm{min}$. 
MccJ25 displays antibacterial activity by inhibiting the RNA polymerase (RNAP) and/or the respiratory chain in the target organism, depending on the bacterial species. ${ }^{23,19,}{ }^{29,30}$ For Salmonella species MccJ25 targets both the RNAP and the cell respiration, however, for E. coli the target is mainly RNAP. Peptides $\mathbf{1}$ and $\mathbf{6}$ most likely do not inhibit RNAP as they are inactive against $E$. coli as well as the lasso structure essential for RNAP inhibition is absent in these peptides. Different structure activity relationship studies of MccJ25 showed that lasso conformation (tail locked in ring) is important in RNAP interaction and inhibition. ${ }^{19,17}$ Thermolysin-cleaved MccJ25 and MccJ25 lacking amino acids 13 to 17 inhibit RNAP transcription suggesting that the unusual lasso ring-tail part of the MccJ25 molecule interacts with RNAP, and blocks the secondary channel of the enzyme. ${ }^{17}$ Whereas, amidation of the Cterminal glycine threaded through the MccJ25 lariat ring specifically blocks the RNAP inhibition. The amidated MccJ25 peptide inhibits cell respiration. ${ }^{19}$

To assess the effect of peptides $\mathbf{1}$ and $\mathbf{6}$ on the inhibition of cell respiration, the oxygen consumption of Salmonella newport in the presence of MccJ25, 1 or $\mathbf{6}$ was assayed. Peptide $\mathbf{1}$ inhibited oxygen consumption, whereas, peptide $\mathbf{6}$ had no effect on oxygen consumption (Figure 5). The findings from this experiment suggest that peptide $\mathbf{1}$ inhibits cell respiration in a manner analogous to MccJ25, and peptide 6 that did not inhibit cell respiration has an alternative mechanism of action. Peptide 1, which lacks the lasso structure, seems to act primarily by inhibition of cell respiration which may also account for its decreased antimicrobial activity compared to MccJ25. 


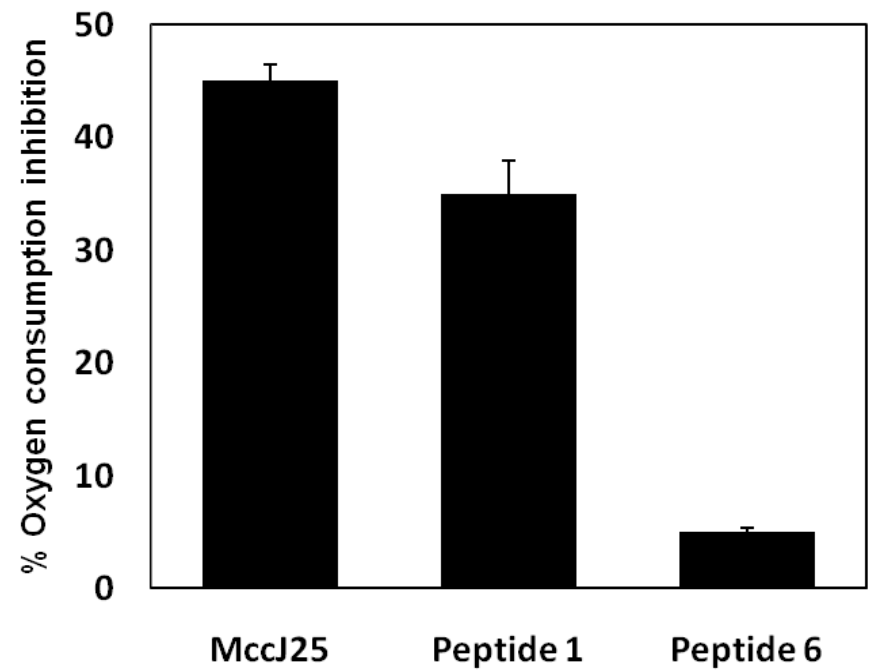

Figure 5. Effects of treatment with MccJ25, peptide 1 and peptide $\mathbf{6}$ on the oxygen consumption. Oxygen consumption of bacteria growing in the presence of peptides was expressed as a percentage of the oxygen concentration of the control culture (without peptides). Data correspond to mean values of three independent experiments. Error bars correspond to the standard deviations.

Finally, the cellular toxicity of MccJ25 and peptides 1-6 was investigated as a lead antibiotic must display low toxicity to mammalian cells. The cytotoxicity of the peptides was evaluated using cell viability MTT assay against two cancer cell lines, MDA-MB-435 and MCF7 cells (Figure S3). MccJ25 and the peptide derivatives displayed very low cytotoxicity (>80\% cell viability) up to the highest concentration tested $(100 \mu \mathrm{M})$. Under similar conditions, a known antitumor agent doxorubicin showed only $20 \%$ cell viability at low concentration $(3 \mu \mathrm{M})$. Peptides 3-4 with charged residues or involving electrostatic interactions displayed slightly higher cytotoxic effect ( $75 \%$ cell viability) compared to the other peptides (> $90 \%$ cell viability). In general, the toxicity values observed were not significant when compared to the known antitumor agents. In this context, it is of particular interest to note that Lopez et al. observed low 
hemolytic activity for MccJ25 against red blood cells. ${ }^{24}$ Although MccJ25 displays antiapoptotic properties with isolated mitochondria, ${ }^{31}$ the low cytotoxicity could be due to its inability to permeate human cells.

To summarize, two active peptides ( $\mathbf{1}$ and $\mathbf{6}$ ) derived from the sequence of MccJ25 are reported. The design strategy used for obtaining active synthetic sequences from the MccJ25 sequence suggests that (i) the folding of the $\mathrm{C}$-terminal tail (in order to constraint the $\mathrm{C}$-terminal tail near the lactam ring) can be achieved with a covalent disulfide bond formation (peptide 1) and (ii) the lactam (or lariat) ring can be substituted with a ring formed by a disulfide bond, however, it may require ring expansion (peptide 6). The $\mathrm{CD}$ and resistance to proteases experiments suggest that the active peptides ( $\mathbf{1}$ and $\mathbf{6})$ do not fold into a lasso conformation, and peptide 1 displays antimicrobial activity by inhibition of target cell respiration. Finally, the synthetic peptide derivatives of MccJ25 display minimal toxicity to mammalian cells and act specifically on bacterial cells.

\section{Experimental Section}

\subsection{Reagents and Equipment}

Wang resin $(0.58 \mathrm{mmol} / \mathrm{g}), \quad \mathrm{N}-\alpha$-Fmoc-amino acids, benzotriazol-L-yl-oxy-tris(dimethylamino)-phosphonium hexafluorophosphate (BOP), and (1-hydroxybenzotriazole) HOBt were purchased from NovaBiochem (San Diego, CA). The side chains of amino acids were protected as follows: tert-butyl $(\mathrm{tBu})$ for serine, threonine, tyrosine, tert-butyl ester ( $\mathrm{OtBu})$ or allyl ester (OAll) for glutamic acid, tert-butoxycarbonyl (Boc) for lysine, trityl (trt) for cysteine and histidine. 2-(1H-7-Azabenzotriazol-1-yl)-1,1,3,3-tetramethyl uronium hexafluorophosphate methanaminium (HATU), 5, 5'-dithiobis-2-nitrobenzoic acid (Ellman's 
reagent), N-methyl morpholine (NMM), triisopropylsilane (TIS), diisopropylethylamine (DIPEA) and trifluoroacetic acid (TFA) were purchased from Aldrich, while piperidine was purchased from Caledon (Canada). All other reagents were purchased from Sigma-Aldrich. All commercial reagents and solvents were analytical grade and were used as received.

RP-HPLC purification and analysis were carried out on a Varian Prostar (210 USA) HPLC system using Vydac semi-preparative C18 (1 x $25 \mathrm{~cm}, 5 \mu \mathrm{m})$, analytical C8 (0.46 x 25 $\mathrm{cm}, 5 \mu \mathrm{m})$ and Agilent preparative $\mathrm{C} 18(21.2 \times 25 \mathrm{~cm}, 7 \mu \mathrm{m})$ columns. Compounds were detected by UV absorption at $220 \mathrm{~nm}$. Mass spectra were recorded on a MALDI Voyager timeof-flight (TOF) spectrometer (Voyager ${ }^{\mathrm{TM}}$ Elite) or on a Waters micromass ZQ. Absorbance of the purple formazan product observed during MTT assay was measured using VERSA max microplate reader (Molecular Devices, Sunnyvale, CA, USA). All the procedures regarding the cell culture maintenance and treatment of cells were carried out in a level II biosafety cabinet.

\subsection{Peptide Synthesis and Purification}

\subsubsection{General procedure}

Stepwise synthesis of peptides 1-6 was done manually on a 0.2-mmol scale of Wang resin (1.0\% DVB cross-linked), following the standard Fmoc solid-phase peptide chemistry as described previously. ${ }^{32}$ Attachment of the first amino acid (5 equivalent) was carried out using 2 , 6 dichlorobenzoylchloride (DCB) in DMF/pyridine mixture. Successive couplings were performed by dissolving an excess (2 equiv) of Fmoc-protected amino acid and Castro reagent (BOP) in presence of $(\mathrm{HOBt})$ to prevent racemization in DMF. Coupling efficiency was monitored using the ninhydrin (Kaiser) test. ${ }^{33}$ The coupling step was repeated (double coupling) if Kaiser test was found positive. In addition, a test cleavage was performed after each five 
residues were coupled, and the desired product was confirmed by MALDI-TOF mass spectrometry. Each peptide was cleaved from the resin using a mixture of $95 \%$ TFA, 5\% triisopropylsilane, 5\% water, for 120 minutes at room temperature with mechanical shaking. The filtrate from the cleavage reactions was collected, combined with TFA washes $(3 \times 2 \mathrm{~min}, 1 \mathrm{~mL})$, and concentrated in vacuum. Cold diethyl ether $(\sim 15 \mathrm{~mL})$ was added to precipitate the crude cleaved peptide. After triturating for $2 \mathrm{~min}$, the peptide was collected upon centrifugation and decantation of the ether. The peptides were then purified using RP-HPLC.

\subsubsection{On resin amide cyclization (Peptides 1 and 2)}

For peptides $\mathbf{1}$ and 2, side chain of Glu was protected as allyl ester (OAll). After complete assembly of the protected peptide on the resin, the side chain protecting group (allyl) of Glu8 (peptide 1) or Glu18 (peptide 2) was first removed. Deprotection of the allyl from carboxyl group was carried out with $\mathrm{Pd}\left(\mathrm{PPh}_{3}\right)_{4}\left(0.08\right.$ equiv) and $\mathrm{PhSiH}_{3}$ (8 equiv) in DCM/DMF (45 mins x 3) under nitrogen. The N-terminus Fmoc group was removed using 20\% piperidine/DMF. The desired lactam linkage was then formed using HATU/DIPEA ( 2 equivalent) as the activating agents for 3 hours in DMF, after which complete cyclization was achieved. It was confirmed using ninhydrin test and MALDI-TOF.

\subsubsection{Oxidative folding and disulfide bond formation}

The purified linear (analogues 3-6) or lactam cyclized peptides (1-2) were dissolved in 50 $\mathrm{mM}$ Tris buffer $(\mathrm{pH} 8.3)$ to a final concentration of $0.1 \mathrm{mM}$. The peptide solution was then treated with $20 \%$ DMSO to assist in oxidation and enhance peptide solubility. The reaction mixture was left stirring overnight at room temperature in an open flask. The oxidation was monitored using Ellman test, ${ }^{34}$ and MALDI-TOF mass analysis. The oxidation was complete in $48 \mathrm{~h}$. A mass spectrum with M-2 indicated loss of two protons and formation of the oxidized 
peptide.

\subsubsection{Peptide purification}

Purification of the peptides was done in two stages. First, the crude linear or lactam cyclized peptides were reconstituted in $30-50 \%$ aqueous acetonitrile and purified on a semipreparative Vydac C18 reversed-phase (RP) HPLC column $(10$ x $250 \mathrm{~mm}, 5 \mu \mathrm{m}$, flow rate $=2$ $\mathrm{mL} / \mathrm{min}$, monitored at $220 \mathrm{~nm}$ ) using different linear gradients of acetonitrile/water or isopropyl alcohol/water $(0.05 \% \mathrm{TFA}, \mathrm{v} / \mathrm{v})$ mixtures. The peptides were oxidized to form the disulfide bond, and were subjected to another RP-HPLC purification using preparative C18 HPLC column $(21.2 \times 250 \mathrm{~mm}, 7 \mu \mathrm{m})$. This purification was essentially done to remove the salts from the reaction mixture. The collected fractions were evaporated on the rotary evaporator followed by lyophilization to obtain the pure peptide. The identity and purity of the peptides were assessed by analytical HPLC (Figure S1) and MALDI-TOF mass spectrometry (Figure S2). The details of the purification methods and the elution time of each peptide are listed in Table S1. In general, pure peptides were obtained with an overall yield of 7-32\% and purity greater than $95 \%$.

\subsection{Microcin J25 Expression and Purification}

The expression and purification of native MccJ25 were performed as described previously with some modifications. ${ }^{12}$ Briefly, the high copy number plasmid pTUC202 (a gift from Rutgers University, USA) which carries the MccJ25 biosynthetic gene cluster was transformed to competent $E$. coli MC4100 cells. The cells were grown in M9 minimal media (2 L) for $24 \mathrm{~h}$ at $37{ }^{\circ} \mathrm{C}$. The culture supernatant was obtained by centrifugation at $4000 \mathrm{~g}$ for 15 min, and then subjected to three successive purification steps. First, the supernatant was applied to flex column filled with XAD16 resin (Aldrich, CA). Followed by elution in two successive 
steps with 30:70 (v/v) and 80:20 (v/v) methanol/water mixtures. Antibacterial assay was used to follow the peptide. MccJ25 found in 80:20 (v/v) methanol/water fraction was next applied to C18 Megabond column (Varian, USA). Three successive elution steps were carried out with 40:60 (v/v), 60:40 (v/v) and 80:20 (v/v) methanol/water mixtures. The 80:20 (v/v) fraction was further purified using C18 RP-HPLC ( $2 \mathrm{~mL} / \mathrm{min}$ flow rate and absorbance monitored at $220 \mathrm{~nm}$ ) under linear gradient from 55-80\% methanol/water $(0.05 \%$ TFA) in $45 \mathrm{~min}$. The concentration of the peptide was determined spectrophotometrically at $278 \mathrm{~nm}$ (molar absorptivity 3340 $\mathrm{M} / \mathrm{L}),{ }^{18}$ and the yield was $2 \mathrm{mg}$.

\subsection{Antimicrobial Activity Assay}

The minimum inhibitory concentrations (MICs) of peptides 1, 6 and MccJ25 were determined by liquid growth inhibition assays in sterile 96-well plate using LB as growth media. $^{22}$ Peptide stock solutions were prepared in 20\% methanol/water (1 mM for MccJ55 and $2.5 \mathrm{mM}$ for $\mathbf{1}$ and $\mathbf{6}$ ), and were serially double diluted in sterile water to give concentrations ranging from 0.01 to $100 \mu \mathrm{M}$ for MccJ25, and 0.5 to $250 \mu \mathrm{M}$ for $\mathbf{1}$ and $\mathbf{6}$. Similar concentration of methanol/water alone without the peptide did not change the growth of bacteria. Bacterial culture $(180 \mu \mathrm{L})$ diluted in LB media were added to the microplate wells to obtain optical density $\left(\mathrm{OD}_{620}\right)$ of $0.02-0.05$, followed by addition of peptide solutions $(20 \mu \mathrm{L})$. Each peptide concentration was tested in triplicate. Control wells contained the peptide in LB media as well as untreated bacterial cells. After incubation of the microplates for $24 \mathrm{~h}$ at $37^{\circ} \mathrm{C}$, the turbidity (OD) was read at $620 \mathrm{~nm}$ on a plate reader. MICs were defined from a growth curve as the lowest peptide concentrations that caused $100 \%$ growth inhibition (0.05 AU). Three representative 
growth curves of microcin $\mathrm{J} 25$, peptide $\mathbf{1}$, and peptide $\mathbf{6}$ obtained using broth assay against Salmonella newport are shown in Figure S4. Standard deviations derived from MIC outcome of three graphs for three repeated experiments were calculated. Concentrations of peptides stocks solutions were determined by absorbance at $280 \mathrm{~nm}$ described previously. ${ }^{35}$ The minimum bactericidal concentration (MBC) was determined from wells showing complete inhibition. An LB agar plate was seeded on the surface with $10 \mu \mathrm{L}$ from each clear well and incubated $(24 \mathrm{~h}$, $37^{\circ} \mathrm{C}$ ). The MBC was defined as the lowest concentration giving no growth on an LB plate afterwards.

\subsection{Circular Dichroism (CD) Spectroscopy}

The CD measurements for the peptides (MccJ25, 1 and 6) were made on an Olis CD spectrometer (Georgia, USA) at $25^{\circ} \mathrm{C}$ in a thermally controlled quartz cell over 190-260 nm. All samples were dissolved in $100 \%$ methanol with a final concentration of $200 \mu \mathrm{M}$. The length of the cuvette was $0.02 \mathrm{~cm}$ and number of scans was set to 10 . Smoothing and correction of the background spectra was performed afterwards. The CD data was normalized and expressed in terms of mean residue ellipticity $\left(\mathrm{deg} \mathrm{cm}^{2} \mathrm{dmol}^{-1}\right)$. CD spectra were analyzed using quantitative curve fitting using the CDPro software analysis program as described previously. ${ }^{36}$

\subsection{Proteolytic Stability}

The enzymatic stability of peptides $\mathbf{1}$ and $\mathbf{6}$ compared to native MccJ25 was evaluated against two digestive enzymes (pepsin and chymotrypsin). The susceptibility of $\mathbf{1}$ and $\mathbf{6}$ for

chymotrypsin cleavage was assessed using spot on lawn method as reported previously. ${ }^{13}$ 
Peptide analogues 1 and $\mathbf{6}(10 \mu \mathrm{L}$ of $200 \mu \mathrm{M})$ were spotted onto LB plates and chymotrypsin solution $(10 \mu \mathrm{L}$ of $0.5 \mathrm{mg} / \mathrm{mL})$ was placed $1 \mathrm{~cm}$ away from the peptide spots. After the drops had dried, the plates were overlaid with $4 \mathrm{~mL}$ of soft agar inoculated with $10^{7}$ cells of a clinical isolate of Salmonella enterica serovar Newport. After overnight incubation at $37{ }^{\circ} \mathrm{C}$, the growth inhibition zones around the drops were recorded. MccJ25 was used as control at $5 \mu \mathrm{M}$ concentration.

Pepsin was dissolved in $0.1 \mathrm{~N} \mathrm{HCl}(4 \% \mathrm{w} / \mathrm{w})$ and then peptide 1 or $\mathbf{6}$ was added (150 $\mu \mathrm{M}) .{ }^{37}$ The solution was incubated at $37{ }^{\circ} \mathrm{C}$ and the degradation was stopped at specific time points $0 \mathrm{~min}, 30 \mathrm{~min}, 1 \mathrm{hr}$, by taking $100 \mu \mathrm{L}$ aliquots into $10 \mu \mathrm{L} 0.1 \mathrm{~N} \mathrm{NaOH}$ containing $70 \%$ methanol to precipitate the enzyme. Sample was centrifuged at $16000 \mathrm{~g}$ for $10 \mathrm{~min}$, and the supernatant containing the cleavage mixture was injected in RP-HPLC. Fractions were collected for mass detection using MALDI-TOF to determine the labile sites. Also fragments were analyzed for antibacterial activity using spot on Lawn as described earlier. For sake of comparison the same experiment was repeated for native MccJ25.

\subsection{Oxygen Consumption Determination}

Oxygen consumption was determined as previously described. ${ }^{38}$ Salmonella newport strain were grown to exponential phase $\left(\mathrm{OD}_{600}\right.$ 0.4-0.5) in LB media. Samples were diluted in $\mathrm{LB}$ media to an $\mathrm{OD}_{600}$ of 0.2 . Cells were then incubated at $37^{\circ} \mathrm{C}$ with either $\mathrm{MccJ} 25(0.25 \mu \mathrm{M})$, $1(25 \mu \mathrm{M})$, or $\mathbf{6}(30 \mu \mathrm{M})$ for $35 \mathrm{~min}$. Thereafter, the average rate of respiration for subsequent 5 min was polographically measured with a $2 \mathrm{~mL}$ Gilson Clarke electrode oxygraph at $37{ }^{\circ} \mathrm{C}$. A control experiment in the absence of peptide was carried out similarly. 


\subsection{Cell Viability Assay}

\subsubsection{Cell lines}

Tumor cell lines used in this study included MDA-MB-435 and MCF7 breast cancer cell lines. MDA-MB-435 cells were cultured in RPMI 1640 medium (Sigma) supplemented with FBS $(10 \%)$, penicillin $(50 \mu \mathrm{g} / \mathrm{mL})$, and streptomycin $(0.05 \mathrm{~g} / \mathrm{mL})$ in a humidified atmosphere $\left(5 \% \mathrm{CO}_{2}\right)$ at $37{ }^{\circ} \mathrm{C}$. MCF7 cells were grown in DMEM media supplemented with same additives as stated above.

\subsubsection{MTT cytotoxicity assay}

The cellular toxicity of MccJ25 and the peptide derivatives was determined by measuring the cell growth inhibition using MTT assay. ${ }^{39}$ Two cancer cell lines were used, MDA-MB-435 and MCF7 cells. Cells were seeded in 96 well plates (Corning Inc., MA, USA) at a concentration of $1 \times 10^{4}$ cells/well $(100 \mu \mathrm{L})$ in complete media and were incubated at $37^{\circ} \mathrm{C}$ in 5\% $\mathrm{CO}_{2}$ atmosphere. After 24 hours, the cells were treated with aseptic samples. Samples (1 $\mathrm{mM}$, MccJ25 and peptides) were prepared in sterile 10\% DMSO/water followed by filtration through $0.2 \mu \mathrm{M}$ filter. The cell culture media were added to the 96 well plate $(180 \mu \mathrm{L} /$ well $)$ followed by addition of peptide sample solution ( $20 \mu \mathrm{L} /$ well), serially double diluted to give concentrations ranging from 0.3125 to $100 \mu \mathrm{M}$. Doxorubicin was used as a positive control and untreated cells were used as a negative control. The plates were incubated for another 24 hours following which the culture media was aspirated and replaced with $100 \mu \mathrm{L}$ MTT solution (5 $\mathrm{mg} / \mathrm{mL}$ in media). The cells were incubated for 3.5 hours to allow interaction with MTT solution. Following incubation, the media was removed by vacuum, and the purple formazan product precipitated in each well was solubilized in DMSO $(100 \mu \mathrm{L})$, and the absorbance was 
measured at $570 \mathrm{~nm}$ using microtitre reader. The percentage cell viability was expressed as the absorbance ratio of cells treated with peptides to untreated cells dissolved in complete media. All experiments were done in triplicate, and the data is presented in the form of mean.

\section{Acknowledgments}

We thank Konstantin Severinov for providing the pTUC202 plasmid and Micheal Stiles, CanBiocin Inc. for helpful discussions and access to the Level 2 Biosafety Laboratory. This work was supported by the Natural Sciences and Engineering Research Council of Canada (NSERC). R.S. is the recipient of the Egyptian Government Scholarship.

\section{Supplementary data}

Characterization of new peptides using reversed phase HPLC and mass spectrometry, details of the bacterial strains, and results from the cell viability MTT assays (Tables S1-S2 and Figures S1-S3). This information can be found, in the online version, at doi:

\section{References}

1. Vincent, P. A.; Morero, R. D. Curr Med Chem 2009, 16, 538.

2. Pavlova, O. A.; Severinov, K. V. Genetika 2006, 42, 1636.

3. Salomon, R. A.; Farias, R. N. J Bacteriol 1992, 174, 7428.

4. Sable, S.; Pons, A. M.; Gendron-Gaillard, S.; Cottenceau, G. Appl Environ Microbiol 2000, 66, 4595 .

5. Wilson, K. A.; Kalkum, M.; Ottesen, J.; Yuzenkova, J.; Chait, B. T.; Landick, R.; Muir, T.; Severinov, K.; Darst, S. A. J Am Chem Soc 2003, 125, 12475. 
6. Rosengren, K. J.; Clark, R. J.; Daly, N. L.; Goransson, U.; Jones, A.; Craik, D. J. J Am Chem Soc 2003, 125, 12464.

7. Bayro, M. J.; Mukhopadhyay, J.; Swapna, G. V. T.; Huang, J. Y.; Ma, L. C.; Sineva, E.; Dawson, P. E.; Montelione, G. T.; Ebright, R. H. J Am Chem Soc 2003, 125, 12382.

8. Delgado, M. A.; Salomón, R. A. Plasmid 2005, 53, 258.

9. Clarke, D. J.; Campopiano, D. J. Org Biomol Chem 2007, 5, 2564.

10. Duquesne, S.; Destoumieux-Garzon, D.; Zirah, S.; Goulard, C.; Peduzzi, J.; Rebuffat, S. Chem Biol 2007, 14, 793.

11. Solbiati, J. O.; Ciaccio, M.; Farias, R. N.; Gonzalez-Pastor, J. E.; Moreno, F.; Salomon, R. A. J Bacteriol 1999, 181, 2659.

12. Blond, A.; Péduzzi, J.; Goulard, C.; Chiuchiolo, M. J.; Barthélémy, M.; Prigent, Y.; Salomón, R. A.; Farías, R. N.; Moreno, F.; Rebuffat, S. Eur J Biochem 1999, 259, 747.

13. Pomares, M. F.; Salomon, R. A.; Pavlova, O.; Severinov, K.; Farias, R.; Vincent, P. A. Appl Environ Microbiol 2009, 75, 5734.

14. Blond, A.; Cheminant, M.; Destoumieux-Garzon, D.; Segalas-Milazzo, I.; Peduzzi, J.; Goulard, C.; Rebuffat, S. Eur J Biochem 2002, 269, 6212.

15. Pan, S. J.; Link, A. J. J Am Chem Soc 2011, 133, 5016.

16. Pavlova, O.; Mukhopadhyay, J.; Sineva, E.; Ebright, R. H.; Severinov, K. J Biol Chem 2008, 283, 25589.

17. Semenova, E.; Yuzenkova, Y.; Peduzzi, J.; Rebuffat, S.; Severinov, K. J Bacteriol 2005, $187,3859$.

18. Bellomio A, V. P., de Arcuri BF, Salomón RA, Morero RD, Farías RN. Biochem Biophys Res Commun 2004, 325(4), 1454. 
19. Vincent, P. A.; Bellomio, A.; de Arcuri, B. F.; Farías, R. N.; Morero, R. D. Biochem Biophys Res Commun 2005, 331, 549.

20. de Cristóbal, R. E.; Solbiati, J. O.; Zenoff, A. M.; Vincent, P. A.; Salomón, R. A.; Yuzenkova, J.; Severinov, K.; Farías, R. N. J Bacteriol 2006, 188, 3324.

21. Ferguson, A. L.; Zhang, S.; Dikiy, I.; Panagiotopoulos, A. Z.; Debenedetti, P. G.; James Link, A. Biophys J 2010, 99, 3056.

22. Montville, C. B.; Lewus, C. B.; Montville, T. J. J Microbiol Methods 1991, 13, 145.

23. Rintoul, M. R.; de Arcuri, B. F.; Salomón, R. A.; Farías, R. N.; Morero, R. D. FEMS Microbiol Lett 2001, 204, 265.

24. Fabian E. Lopez, P. A. V., Ana M. Zenoff, Rau' 1 A. Salomo'n and Ricardo N. Farı'as. J Antimicrob Chemother 2007, 59, 676.

25. Blond, A.; Cheminant, M.; Segalas-Milazzo, I.; Peduzzi, J.; Barthelemy, M.; Goulard, C.; Salomon, R.; Moreno, F.; Farias, R.; Rebuffat, S. Eur J Biochem 2001, 268, 2124.

26. Liu, S.; Zhou, L.; Li, J.; Suresh, A.; Verma, C.; Foo, Y. H.; Yap, E. P.; Tan, D. T.; Beuerman, R. W. Chembiochem 2008, 9, 964.

27. Woody, R. W. Biopolymers 1978, 17, 1451.

28. Sreerama, N.; Woody, R. W. Anal Biochem 2000, 287, 252.

29. Delgado, M. A.; Rintoul, M. R.; Farias, R. N.; Salomon, R. A. J Bacteriol 2001, 183, 4543.

30. Yuzenkova, J.; Delgado, M.; Nechaev, S.; Savalia, D.; Epshtein, V.; Artsimovitch, I.; Mooney, R. A.; Landick, R.; Farias, R. N.; Salomon, R.; Severinov, K. J Biol Chem 2002, $277,50867$.

31. Chirou, M. N.; Bellomio, A.; Dupuy, F.; Arcuri, B.; Minahk, C.; Morero, R. FEBS J 2008, $275,4088$. 
32. Kaur, K.; Andrew, L. C.; Wishart, D. S.; Vederas, J. C. Biochemistry 2004, 43, 9009.

33. Kaiser E, Colescott, R. L.; Bossinger, C.D.; Cook, P. I. Anal Biochem 1970, 34, 595.

34. Ellman, G. L. Arch Biochem Biophys 1959 82(1), 70.

35. Layne, E. Methods Enzymol 1957, 3, 447.

36. Sreerama, N.; Venyaminov, S. Y.; Woody, R. W. Anal Biochem 2000, 287, 243.

37. Bryant, K. J.; Read, L. C.; Forsberg, G.; Wallace, J. C. Growth Factors 1996, 13, 261.

38. Chalon, M. C.; Bellomio, A.; Solbiati, J. O.; Morero, R. D.; Farias, R. N.; Vincent, P. A. FEMS Microbiol Lett 2009, 300, 90.

39. van de Loosdrecht AA, B. R., Ossenkoppele GJ, Broekhoven MG, Langenhuijsen MM. J Immunol Methods 1994, 174, 311. 
Graphical Abstract

\begin{tabular}{|c|c|c|}
\hline Peptide & & $\begin{array}{c}\mathrm{MIC}(\mu \mathrm{M}) \\
\text { against } S \text {. newport }\end{array}$ \\
\hline$\overbrace{\mathbf{1}}^{\text {GGAGHVPEYFVGIGTPISFYG }}$ & MccJ25 & 0.5 \\
\hline$\underset{\mathbf{G G A C H V P E Y F V G I G T P I S F C ~}}{{ }_{\mathbf{8}}}$ & 1 & 25 \\
\hline${ }_{1}^{\text {CGAGFHVPCCYFVRGTPISFYG }}$ & 6 & 30 \\
\hline
\end{tabular}

